\title{
The importance of being solid for a ground glass opacity of the lung
}

\author{
Luca Bertolaccini ${ }^{1}$, Lorenzo Spaggiari ${ }^{1,2}$ \\ ${ }^{1}$ Department of Thoracic Surgery, IEO, European Institute of Oncology IRCCS, Milan, Italy; ${ }^{2}$ Department of Oncology and Hemato-Oncology, \\ University of Milan, Milan, Italy \\ Correspondence to: Luca Bertolaccini, MD, PhD, FCCP. Division of Thoracic Surgery, IEO, European Institute of Oncology IRCCS, Via Ripamonti \\ 435 - 20141 Milan, Italy. Email: luca.bertolaccini@gmail.com.
}

Submitted Apr 30, 2021. Accepted for publication May 26, 2021.

doi: $10.21037 / \mathrm{atm}-21-1270$

View this article at: http://dx.doi.org/10.21037/atm-21-1270

"He who lives by the crystal ball soon learns to eat ground glass."-Edgar Russel Fiedler (1929-2003)

Low-dose computed tomography (LDCT) has revolutionised the worldwide arena of lung cancer screening over the past several decades. Due to the increase of screening with LDCT, a growing number of ground-glass opacity (GGO) lesions of the lung are discovered. The pattern of a GGO at computed tomography (CT) scan should encounter four key criteria:

(I) Areas of hazy and amorphous increased lung attenuation;

(II) No obscuration of the vascular markings and underlying bronchial walls;

(III) Identification of high-resolution CT sections;

(IV) Identification with wide window settings (1).

Besides, the GGO was also categorised as pure GGO and part-solid nodules (2). Several parameters could estimate the solid proportion of GGO as the consolidation-to-tumour ratio (ratio of the size of solid portion to total size in lung window of the CT scan) or the tumour disappearance ratio (difference from the unity between the ratio of the size of the solid portion in the mediastinal window of CT scan and total tumour size in the lung window of CT scan) (3).

Nevertheless, the knowledge and managing of GGO in the lung cancer field yet need investigation. Due to the limited evidence from randomised controlled trials regarding GGO managements, the worldwide debate will probably continue for a while (4).

Classical lung cancer was prevalent among elderly patients, men, and smokers. On the contrary, the average age at diagnosis decreased. Simultaneously, in the past two decades, the non-smoking amount was significantly augmented, demonstrating that most GGO adenocarcinoma patients are being spotted from the conventionally low-risk groups of non-smokers young females, making it essential to reassess the risk assessment and the current criteria for lung cancer screening (4).

Therefore, we have read with interest the paper of Wang et al. from the Sichuan University $(\mathrm{CN})$, recently published in the Annals of Translational Medicine, with an in-depth analysis of a large cohort of resected stage I nonsmall-cell lung cancer (NSCLC) subdivided into three subgroups (pure-GGO, part-solid, and pure solid). Authors demonstrated that the GGO component is a predictor of favourable prognosis for stage I NSCLC, supporting the suggestion that the next edition of the tumour-nodemetastasis (TNM) classification should contemplate the GGO parts as a new T descriptor (5).

As reported by the authors, this study also had several limitations like the retrospective nature. Retrospective cohort studies are generally created from collected databases of healthcare records. Selection bias would have happened if the cohort selected was not representative of all possible population patients. Misunderstanding often occurs in the meaning of the population term in statistics, probably because it has a different connotation from its general every day (used in a geographical sense). For Statistics, the population is habitually regarded as an infinite group of people. Hence, selection bias should have been minimised (6).

The study of Wang et al. was also built on singleinstitution and Chinese people with a high incidence of the never-smokers and lung adenocarcinoma patients (5). Besides, due to cheap and easy access to LDCT in China, 


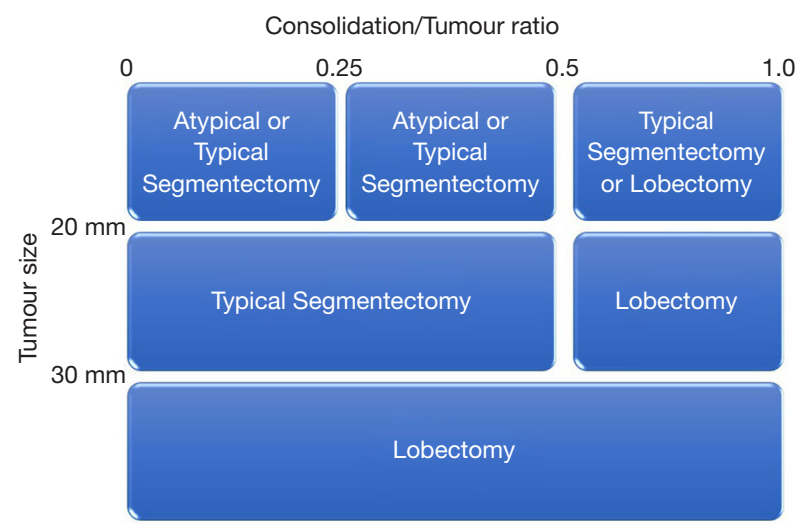

Figure 1 Strategies for stage I lung cancer decided by considering the maximum tumour dimension and consolidation tumour ratio (9).

many companies proposed LDCT screening as a regular health check-up to their workers. Therefore, LDCT screening is prevalent in the traditionally high-risk and lowrisk populations (4).

The cohort sample size of the study from Wang et al. may also be limited (5). Cohort studies present advantages in evaluating numerous exposure factors and outcomes (primary and secondary). These studies can be used to expected outcomes and unusual exposure factors. Usually, due to the cohort follow-up time, these studies are relatively expensive. If a statistical association between exposure and outcome was found, the experimental is requested to validate the hypothesis (7). Hence, a more significant population from multinational centres in randomised controlled trials might be necessary to confirm the prognostic value of the GGO components (5).

No information was reported in the paper about the surgical operation (e.g., atypical segmentectomy versus typical segmentectomy versus lobectomy). Since 1960, lobectomy is the gold standard even for small-sized lung cancers, and typical segmentectomy could be accepted for patients unfit for lobectomy. In patients with clinical stage IA NSCLC who underwent sublobar resection, a propensity score-matched analysis showed that cumulative incidence of recurring after segmentectomy was significantly lower than that after atypical segmentectomy. Although segmentectomy appears to be better at atypical segmentectomy from the perspective of cancer recurrence and the cumulative incidences of death without recurrence, the procedures were not statistically different. Therefore, the surgical procedures should be carefully designated, particularly in patients contemplated as high risk for lobectomy (8).
Several strategies for the right surgical approach considered the maximum tumour dimension and consolidation tumour ratio. Small and less-invasive lung cancers were treatable with sublobar resection instead of lobectomy (Figure 1). Sublobar resection with radical surgical margin presented an adequate local control and disease-free survival for N0 NSCLC staged by CT with three or fewer peripheral lesions $\leq 2.0 \mathrm{~cm}$ with a consolidation tumour ratio $\leq 0.25$ (9). A typical segmentectomy is more advantageous from an oncological perspective than an atypical segmentectomy because it allows nodal dissection at the hilum.

Consequently, precise staging should be contemplated in any conclusion regarding the suitability of segmentectomy or lobectomy. After the results of the prospective randomised phase III trials on segmentectomy will be validated, segmentectomy could probably become the benchmark procedure for early-stage and small peripheral NSCLC with an equivalent curability and less toxicity than lobectomy. Therefore, surgeons will need to master segmentectomy as the frequency of detecting small lung cancers increases.

Conversely, tumour location is likewise a critical factor that influences the choice of surgical procedure. A centrally located tumour that is tricky to treat by wedge resection can usually be tackled by segmentectomy with an acceptable surgical margin. However, some tumours remain difficult to resect by segmentectomy with a sufficient surgical margin, such as those situated around the lobar bronchus root (10).

Thus, the first stage of selecting lobectomy or segmentectomy is the assessment of the whole tumour size and tumour location on preoperative CT images. Surgeons might be conscious of complex segmentectomy in terms of tumour location for segmentectomy. Segmentectomy that establishes one linear intersegmental plane with a comparatively uncomplicated procedure can be deemed simple: resectioning the right sixth left upper-division or lingular segments. Segmentectomy that generates several complicated intersegmental planes can be judged complex as the resection of the right I, left IX, right I and III, and left IX and X segments even if types and rates of postoperative complications do not significantly change (10).

In conclusion, it is crucial to explain the adenocarcinoma expressed as GGO by LDCT imaging cautiously to prevent unnecessary overdiagnosis and overtreatment. It is also exceedingly suggested that the multidisciplinary team experts examine the imaging report standard for negative/ positive pure GGO to minimise further the possibilities of overdiagnoses and overtreatment (11). 


\section{Acknowledgments}

Funding: This work was partially supported by the Italian Ministry of Health with Ricerca Corrente and $5 \times 1000$ funds.

\section{Footnote}

Provenance and Peer Review: This article was commissioned by the editorial office, Annals of Translational Medicine. The article did not undergo external peer review.

Conflicts of Interest: Both authors have completed the ICMJE uniform disclosure form (available at http://dx.doi. org/10.21037/atm-21-1270). The authors have no conflicts of interest to declare.

Ethical Statement: The authors are accountable for all aspects of the work in ensuring that questions related to the accuracy or integrity of any part of the work are appropriately investigated and resolved.

Open Access Statement: This is an Open Access article distributed in accordance with the Creative Commons Attribution-NonCommercial-NoDerivs 4.0 International License (CC BY-NC-ND 4.0), which permits the noncommercial replication and distribution of the article with the strict proviso that no changes or edits are made and the original work is properly cited (including links to both the formal publication through the relevant DOI and the license). See: https://creativecommons.org/licenses/by-nc-nd/4.0/.

\section{References}

1. Remy-Jardin M, Remy J, Giraud F, et al. Computed tomography assessment of ground-glass opacity: semiology and significance. J Thorac Imaging 1993;8:249-64.

2. Hattori A, Matsunaga T, Takamochi K, et al. Neither Maximum Tumor Size nor Solid Component Size Is

Cite this article as: Bertolaccini L, Spaggiari L. The importance of being solid for a ground glass opacity of the lung. Ann Transl Med 2021;9(13):1043. doi: 10.21037/atm-21-1270
Prognostic in Part-Solid Lung Cancer: Impact of Tumor Size Should Be Applied Exclusively to Solid Lung Cancer. Ann Thorac Surg 2016;102:407-15.

3. Kim H, Goo JM, Kim YT, et al. Consolidation-to-tumor ratio and tumor disappearance ratio are not independent prognostic factors for the patients with resected lung adenocarcinomas. Lung Cancer 2019;137:123-8.

4. Zhang Y, Fu F, Chen H. Management of Ground-Glass Opacities in the Lung Cancer Spectrum. Ann Thorac Surg 2020;110:1796-804.

5. Wang C, Wu Y, Li J, et al. Distinct clinicopathologic factors and prognosis based on the presence of groundglass opacity components in patients with resected stage I non-small cell lung cancer. Ann Transl Med 2020;8:1133.

6. Sedgwick P. Retrospective cohort studies: advantages and disadvantages. BMJ 2014;348:g1072.

7. Camargo LMA, Silva RPM, Meneguetti DUDO. Research methodology topics: Cohort studies or prospective and retrospective cohort studies. J Hum Growth Dev 2019;29:433-6.

8. Tsutani Y, Handa Y, Shimada Y, et al. Comparison of cancer control between segmentectomy and wedge resection in patients with clinical stage IA non-small cell lung cancer. J Thorac Cardiovasc Surg 2020. [Epub ahead of print]. doi: 10.1016/j.jtcvs.2020.10.024.

9. Suzuki K, Watanabe SI, Wakabayashi M, et al. A singlearm study of sublobar resection for ground-glass opacity dominant peripheral lung cancer. J Thorac Cardiovasc Surg 2020. [Epub ahead of print]. doi: 10.1016/ j.jtcvs.2020.09.146.

10. Mimae T, Okada $M$. Are segmentectomy and lobectomy comparable in terms of curative intent for early stage non-small cell lung cancer? Gen Thorac Cardiovasc Surg 2020;68:703-6.

11. Chen $\mathrm{KN}$. The diagnosis and treatment of lung cancer presented as ground-glass nodule. Gen Thorac Cardiovasc Surg 2020;68:697-702. 